\title{
DESARROLLO Y ANÁLISIS DE LAS POLÍTICAS EDUCACIONALES EN GESTIÓN ESCOLAR IMPLEMENTADAS EN CHILE, PERÍODO I990 - 2006
}

\section{Gustavo González García}

\section{RESUMEN:}

Este artículo describe y analiza, desde una perspectiva general y sintetizada, el desarrollo en el tiempo de las políticas educacionales en Gestión Escolar implementadas en Chile durante el período 1990-2006. En particular, describe el proceso por el cual la Gestión Escolar, de tener un valor auxiliar en el diseño de las políticas durante los noventa, llega a ser una de las estrategias más relevantes - desde el año 2003- para elevar la calidad del sistema escolar chileno.

Palabras clave: Políticas Educacionales; Gestión Escolar; Sistema Escolar.

\section{DEVELOPMENT AND ANALYSIS OF EDUCATIONAL POLICIES ON SCHOOL MANAGEMENT IMPLEMENTED IN CHILE DURING THE I990-2006 PERIOD}

\begin{abstract}
:
This paper describes and analyzes, from a general and synthesized perspective, the development in the time of the educational policies in School Management implemented in Chile during the period 1990-2006. In particular, describes the process by which the School Management, from having an auxiliary value in the design of the policies during the nineties, become in one of the most relevant strategies -form the year 2003- to rise Chilean School system quality.
\end{abstract}

Keywords: Educational Policies, School Management, School System.

* Académico del Centro de Investigaciones Pedagógicas, Universidad Arturo Prat, Victoria. Candidato a Doctor en Ciencias de la Educación, Pontificia Universidad Católica de Chile. Email: gdgonzal@uc.cl 
Desarrollo y análisis de las políticas educacionales en gestión escolar implementadas en Chile, período 1990 - 2006 - Gustavo González García

\section{Introducción}

El concepto de gestión escolar puede ser definido de variadas maneras. No siendo el propósito de este artículo la teorización del concepto, entenderemos por gestión escolar definiciones como la aportada por Gairín (1996), quien reconoce por gestión escolar aquellos procesos y actuaciones ejecutados, coordinados y evaluados al interior de la organización escolar, con vistas a conseguir la mejor realización de un proyecto educativo.

Coincidente con esta definición, Hornkohl (2003), como autoridad del Ministerio de Educación Chileno (MINEDUC) en el año 2003, entendía por gestión escolar:

"el proceso mediador que articula y coordina las diversas acciones que emprende la organización escolar, permitiendo la generación de condiciones apropiadas para la principal tarea que debe desarrollar la escuela o liceo: el mejoramiento de los aprendizajes de los estudiantes cbilenos, sus procesos formativos y los resultados institucionales".

Lo que interesa dejar en claro tras las referencias, es que aquel ámbito de acción relacionado con los procesos que ocurren en el espacio organizacional de la escuela -que está inequívocamente relacionado con logros de aprendizaje obtenidos en el aula- es un ámbito que ha interesado al desarrollo científico y académico educacional desde hace mucho tiempo, pero que en las últimas décadas ha tenido un mayor impacto en la producción científica chilena y en los impulsos de mejoramiento sobre el sistema escolar nacional. Esto es producto de variados factores, entre otros; los procesos de descentralización educacional; el efecto de los movimientos y modelos teóricos de efectividad escolar y cambio educativo; el desarrollo de los sistemas de evaluación y sistemas de accountability, que buscan evaluar calidad educativa y responsabilizar por logros; etc. (ver Alvariño, et al., 2000).

I Discurso de la Subsecretaria de Educación María Ariadna Hornkohl, en el Seminario Internacional "Gestión Escolar y Liderazgo Directivo", realizado los días I y 2 de abril de 2003 en dependencias del Centro de Extensión de la P. Universidad Católica, en Santiago. 
El objetivo de este artículo es describir la consideración que ha tenido la gestión escolar en el diseño y desarrollo de las políticas educacionales chilenas, período I990-2006. También se busca analizar y entregar pistas sobre el impacto que estas políticas han tenido en su momento y que han condicionado el desarrollo de las políticas siguientes.

El artículo se divide en la descripción y análisis de dos períodos; durante los noventa y a partir del año 2000.

\title{
I. La Gestión Escolar y las Políticas Educacionales durante los Noventa
}

\author{
a) Período Inicial, I990-I993: valoración intuitiva del \\ efecto Gestión Escolar en los resultados de las políticas \\ educacionales.
}

La gestión escolar o educativa no fue incorporada como una variable de intervención, con rango particular, en el diseño de las políticas educacionales del período 1990-1993. Si bien existió en el período una concepción de que las escuelas eran las unidades de intervención y transformación de los cambios educativos que se planteaban (García-Huidobro y Sotomayor, 2003) y que además el diseño de las políticas se hacía sobre escuelas que presentaban características y exigencias especiales de intervención (las con más dificultades por sus resultados y contextos socioeconómicos). Sin embargo, la centralidad de programas de mejoramiento como el Programa de las 900 Escuelas (P-900), se dirigía hacia el cambio de las prácticas pedagógicas en el aula, a través de la mejoría de los resultados escolares en lenguaje y matemáticas; con estrategias específicas de perfeccionamiento docente (Talleres de profesores) y atención a los niños con retraso escolar (Talleres de Aprendizaje).

Posteriormente, en el caso del P-900, fue el análisis crítico de la eficacia en el logro que estaban teniendo los objetivos del Programa, en sus primeros años, lo que llevo a la conclusión de la necesidad de 
elevar la mirada hacia el ámbito de la escuela, apoyando la gestión de éstas (Larraín, 2002). Al respecto, las primeras evaluaciones del P-900 (Flip y otros, I99I), sugerían fortalecer la identidad de cada escuela; mejorar el clima organizacional; las relaciones entre profesores, alumnos y apoderados y conseguir el compromiso de los directores con los objetivos del Programa, considerando perfeccionamiento especial para éstos.

\section{b) Período 1993-2000: Fortalecimiento de la gestión escolar y la implementación de los primeros modelos.}

En el contexto de favorecer los logros del P-900, se implementa en I 993 un primer proyecto de Fortalecimiento a la Gestión Escolar desarrollado por el MINEDUC, con la asesoría de la Facultad de Educación de la Pontificia Universidad Católica de Chile y del Centro de Investigación y Desarrollo de la Educación (CIDE). El objetivo principal fue organizar y articular el trabajo de sostenedores, supervisores técnico-pedagógicos y directores a través de la creación de organismos e instrumentos de planificación, como los Equipos de Gestión Comunal (EGC, reuniendo a los tres actores señalados), la conformación de Equipos de Gestión Escolar (EGE) en los establecimientos y el diseño de Proyectos Educativos Institucionales (PEI).

Este primer proyecto de fortalecimiento de la gestión escolar estaba guiado por conceptos de planificación estratégica y buscaba generar sistemas de gestión participativos y colaborativos de los asuntos institucionales, bajo la conducción del Director de la escuela. A partir de 1998 se reformula el modelo de fortalecimiento de la gestión, la nueva etapa es asesorada por la Universidad Academia de Humanismo Cristiano y por el Programa Interdisciplinario de Investigaciones en Educación (PIIE); se trata de una etapa de institucionalización de la línea de gestión educativa del P-900, donde se pasa de un enfoque de gestión centrado en la planificación estratégica, a otro de planeamiento interactivo o estratégico-situacional (Larraín, 2002). Dentro de los aspectos centrales de esta nueva fase se encuentran 
la promoción de instancias de negociación y acuerdos, sobre la base de compromisos de gestión, entre el Director, el sostenedor y el MINEDUC -que funciona a través de los Departamentos Provinciales de Educación (DEPROV), representados por el supervisor técnico pedagógico, designado a cada escuela-. También se promueve la reformulación de los PEI y la inclusión de planes anuales de acción que lo operacionalicen; estimular la conformación más amplia de los equipos de gestión; fortalecer el liderazgo pedagógico de los docentes directivos y desarrollar mecanismos de evaluación y seguimiento institucional.

c) El Impacto de la Política en gestión educacional durante los noventa: análisis de las evaluaciones académicas.

Evaluaciones del impacto del diseño ministerial en la gestión escolar de los establecimientos favorecidos por los programas de mejoramiento, concluyen que hay aspectos positivos, especialmente en la implementación de prácticas formales, pero existen dificultades importantes en la profundidad y sentido de los cambios planificados.

En relación a los instrumentos claves del diseño ministerial, en materia de gestión escolar en los noventa; la constitución y fortalecimiento de los Equipos de Gestión (EGE), la planificación institucional y su concreción -Proyecto Educativo Institucional (PEI), Planes Anuales de Acción, Compromisos de Gestión-, una evaluación del P-900 (Asesorías para el Desarrollo, 2000), señala que existen avances en estos indicadores formales, tales como que del $92 \%$ de las escuelas que estaban en el P-900 en la fase 1997-2000 y el 79\% que estuvieron en el período anterior, contaban con un EGE y un $65 \%$ con un PEI implementado, sin embargo, el estudio también concluye que estos instrumentos no eran percibidos como productos vivos, presentes en las prácticas escolares cotidianas, orientando el proceso continuo de decisiones en los establecimientos.

Este mismo estudio de Asesorías para el Desarrollo (idem), observó más avances en la instalación de algunas prácticas de gestión 
Desarrollo y análisis de las políticas educacionales en gestión escolar implementadas en Chile, período 1990 - 2006 - Gustavo González García

participativas, orientadas al mejoramiento del clima organizacional y a la satisfacción institucional, como cambios en el liderazgo de directores; que estimulan el trabajo cooperativo, identifican proyectos a seguir, gestionan recursos externos y estimulan las relaciones con la comunidad. Por otra parte, un porcentaje importante de los docentes reconocían tener mayor capacidad de decisión en asuntos como el PEI, innovaciones curriculares y proyectos de mejoramiento. El estudio termina concluyendo, que el impacto en la gestión escolar no fue el esperado en aspectos centrales, como el no haber generado en las instituciones una imagen de cómo debiera ser la gestión educativa, definir los responsables para cada ámbito y la poca capacidad para hacer funcionar los EGE e implementar los PEI. En definitiva, se planteaba que los establecimientos no fueron capaces de implementar los planeamientos institucionales que formalmente elaboraban; definiendo compromisos, conduciendo y exigiéndolos.

Otra evaluación del P-900 para el período 2001-2003, de la Universidad Católica (Martinic y otros, 2005), llega a conclusiones más positivas en cuanto a la buena evaluación de la gestión directiva en los mismos ámbitos señalados anteriormente y en la significativa participación de los docentes en los procesos de toma de decisiones pedagógicas, menos en los financieros.

En relación al impacto en los indicadores formales, el estudio plantea que éstos se instalaron formalmente y que, en general, son bien valorados como instrumentos de gestión, pero que no concitan la aprobación total, con respecto a lo que se espera de ellos. Al respecto, el 52,8\% de los profesores estudiados sostenían que el EGE tiene un rol fundamental en la gestión educativa, pero el 21,8\% estimaba que sólo tenía una participación acotada en algunos temas, el I8,4\% señalaba que participaba eventualmente en casos específicos y un 6,9\% sostenía que el EGE se conformó pero nunca funcionó.

En relación al PEI, el estudio concluía que en el $69 \%$ de los establecimientos se implementó. Los docentes en un $97,3 \%$ señalaban que en el PEI se definía un plan de acción anual, el 94,3\% sostenía que 
éste se evaluaba anualmente y el $88,2 \%$ estimaba que sus resultados se comunicaron a la comunidad escolar.

\section{d) El balance de las políticas educacionales en gestión escolar durante los noventa.}

Un análisis descriptivo y crítico de las políticas educacionales, sobre todo de los estudios de los Programas de mejoramiento y las evaluaciones de impacto de éstos, permite concluir ciertas ideas sobre cómo las políticas influyeron en la forma de gestionar las escuelas y liceos.

En primer lugar, la gestión escolar no fue considerada desde sus inicios como política educacional con rango propio, pero tempranamente se le tuvo en cuenta y se le valoró como una variable importante para el éxito de los Programas de Mejoramiento.

Asociada a los Programas, la gestión escolar tuvo un ámbito de implementación reducido a los establecimientos que fueron favorecidos por aquellos y por la política de focalización, es decir, en una población de centros escolares que, al final de la década, no era superior al I0\% del sistema.

Las ideas y teorías que inspiran los diseños de fortalecimiento de la gestión escolar en el período, provienen de la planificación estratégica, círculos de calidad, la teoría de las organizaciones, la administración educacional y algunos elementos del management moderno. El Manual para Equipos de Gestión Escolar (Astudillo y otros, 1996), documento oficial que orientó el trabajo de gestión escolar en los establecimientos del P-900, representa claramente este marco teórico. El Manual conceptualiza, intenciona y guía contenidos de gestión escolar; como la noción de la Escuela como una organización que tiene identidad, autonomía y proyecto propio (PEI); la necesidad de planificación de metas, seguimiento y evaluación de las tareas; la importancia del trabajo en equipo, la gestión participativa, el mejoramiento del clima de trabajo y la resolución 
Desarrollo y análisis de las políticas educacionales en gestión escolar implementadas en Chile, período 1990 - 2006 - Gustavo González García

de conflictos, planificación y ejecución de reuniones efectivas, entre otros contenidos.

Aquí predomina una teoría basada en la gestión de las organizaciones en general y no se encuentran contenidos de organización y gestión escolar propiamente tal. Entendiendo por esto, una caracterización teóricamente más precisa de la organización escolar a nivel, por ejemplo, de la identificación de elementos que son propios de la escuela y diferentes de otras organizaciones; como sus particulares ámbitos de funcionamiento, procesos de calidad específicos, indicadores educativos de eficacia, modelos de evaluación educacional, entre otros. Elementos ya presentes, a nivel del estado de desarrollo de movimientos teóricos como la efectividad escolar, el cambio educativo y mejoramiento escolar. Movimientos que hacia los noventa, ya disponían de cúmulos de conocimientos acerca de lo que es una buena escuela, como funciona y cuáles son los indicadores de su eficacia.

En síntesis, la gestión escolar concebida en el diseño de las políticas durante el período, se basa en ciertas ideas que conciben a la escuela como unidad de mejora y como contexto de acción, pero en el marco de la teoría organizacional general y gestión de calidad, que se aplica a lo educativo.

Esta preliminar idea de la escuela como organización, que tiene una identidad propia y puede gestionar participativamente la calidad, se basó en dos fundamentos; uno político, el de profundizar y democratizar la descentralización educacional -en un período particular de la historia política del país, de transición a la democracia-; y un fundamento teórico, aquel que planteaba que la escuela puede y debe afectar los resultados escolares de los alumnos. Probablemente, en esto último, están influyendo los primeros estudios realizados para la realidad escolar chilena, sobre la influencia de la escuela en los resultados de aprendizaje (Flip y otros, I98I/I982; Himmel y otros, 1984).

En relación a las estrategias de diseño e implementación de las políticas de gestión escolar durante los noventa, el diseño es elaborado 
en sus contenidos centrales por los equipos ministeriales de los Programas de Mejoramiento, pero una vez que estos equipos definen prioridades y necesidades, buscan alianzas técnicas con especialistas de universidades y centros de estudios, para elaborar los diseños definitivos.

La implementación de las políticas en gestión está en manos de la supervisión técnicos pedagógica, por ser ésta la que implementa la política educacional y los programas de mejoramiento. Son los supervisores los que se capacitan en el modelo de fortalecimiento de la gestión, para que asesoren y propicien la formulación de PEI, acuerden metas de gestión y supervisen la aplicación de los dispositivos de gestión, como el Manual de Equipos de Gestión.

\section{La Gestión Escolar y las Políticas Educacionales a partir del 2000}

a) Período 2000-2003: fortalecimiento del Modelo de Gestión Escolar a partir de la concepción de Organización Escolar.

En los primeros años del actual siglo, el diagnóstico que se derivaba de los análisis ministeriales e informes de evaluación de los programas de mejoramiento, en el ámbito de lo implementado en gestión escolar, era que había que avanzar mejorando la apropiación significativa del modelo de fortalecimiento de la gestión. Estaba la idea de que el PEI, los EGE, los sistemas de calidad basados en la planificación estratégica, eran dispositivos instalados formalmente en los establecimientos, exigidos por la normativa ministerial (Larraín, 2002), pero que no habían sido incorporados a la cultura escolar.

En los dos primeros años del siglo, para mejorar este diagnóstico, el MINEDUC elabora y difunde instrumentos que potencian el modelo de gestión escolar, incorporando una concepción más singular y, a la vez, más operativa, de los establecimientos como organizaciones particulares, con distintas dimensiones de intervención. Esta visión 
Desarrollo y análisis de las políticas educacionales en gestión escolar implementadas en Chile, período 1990 - 2006 - Gustavo González García

más clara de la organización escolar se ve reflejada en distintos documentos, elaborados o promovidos oficialmente, que aparecen desde fines de los noventa: El Proyecto Educativo Institucional como berramienta de construcción de identidad (Lavín, del Solar y Padilla, 1997), la Guía de Autoevaluación de la Escuela (Mineduc, 2000), y en el documento Hacia una Gestión Autónoma y centrada en lo Educativo. Propuesta del Programa de las 900 Escuelas (Larraín, 2002b). Estos dispositivos comienzan a plantear que la institución educativa es una organización original, donde se pueden identificar distintas dimensiones de acción, ámbitos que se pueden intervenir por separado, pero teniendo en cuenta que todas éstos deben articularse en torno al proyecto educativo. Se identifican dimensiones; como la pedagógica-curricular, la organizativa-operacional, la administrativa-financiera, la comunitaria, la convivencial. Se siguen planteando elementos de planificación estratégica y se comienzan a plantear lógicas de mejoramiento en la identificación de los procesos de calidad: autoevaluación; planificación anual, seguimiento y evaluación, cuenta pública.

A partir del 2000, todas las escuelas del P-900, al decir de una de sus diseñadoras, "desarrollan una autoevaluación en diversas dimensiones y definen metas y acciones estratégicas en planes anuales, cuya evaluación es compartida en las jornadas de gestión con otras escuelas y, en algunos casos, son presentadas a los sostenedores y supervisores para negociar sus apoyos para el año siguiente" (Larraín, 2002, p.I62).

\section{b) Período 2003-2006: La Gestión Escolar adquiere estatus propio y valor estratégico para la Política educacional.}

Hacia el 2003, la evaluación que se hace de todas las políticas educacionales iniciadas en 1990, está en un contexto vulnerable. Esto debido a la opinión pública sobre lo que ha sido el talón de Aquiles de la Reforma: el débil impacto de las políticas en los resultados de aprendizaje de los establecimientos con financiamiento público, donde se educa la mayoría de la población. 
La urgencia y presión por los resultados provoca una situación que comienza a concebir un estatus más privilegiado de la gestión escolar, para dejar de ser una estrategia auxiliar de los programas de mejoramiento y pasar a tener rango propio de política educacional. Esto se refuerza porque, en paralelo, los anteriores Programas de Mejoramiento empiezan a perder respaldo. Éstos terminan o se reconvierten, ante la crítica por su orientación de arriba hacia abajo, desde la mirada de las escuelas (Raczynski y otros, 2002), que terminan explicando su débil impacto en la realidad escolar.

No es extraño, entonces, que la gestión escolar comience a ser revalorada, como una variable con alta capacidad de solucionar un contexto desfavorable: la resistencia del sistema escolar a elevar sus resultados. Esto se refuerza porque, más generalizadamente, la efectividad escolar está impactando teóricamente en la discusión académica y pública, en particular por su contribución acerca de cómo aquellos procesos que se generan a nivel de la escuela afectan, empeoran o favorecen, los aprendizajes de los alumnos, a partir de sus condiciones de entrada (sociales y culturales). Por lo tanto la relación gestión escolar-resultados de aprendizaje resulta evidente.

El Informe de Revisión de Políticas Educacionales en los noventa (OCDE, 2004), también tiende a reforzar la idea que la gestión escolar es una de las variables claves en torno a solucionar los problemas educativos del país. Cuando identifica que uno de los tres problemas fundamentales del sistema escolar chileno, pasa por una debilidad de gestión, relacionada con la falta de supervisión educativa, cuando ésta ha establecido un nexo débil entre los esfuerzos y objetivos planteados desde la política educativa, con las acciones emprendidas y los resultados conseguidos en las escuelas. La supervisión no sólo entendida por aquella de carácter pública que ejerce el MINEDUC, sino la que surge de la articulación en la entrega de apoyo técnico y exigencia a las escuelas, entre los directivos y su liderazgo interno, el Mineduc, los sostenedores y las Universidades.

Todos estos antecedentes explican que a partir del año 2003, la gestión escolar pasa a tomar un papel central de la política educacional, 
Desarrollo y análisis de las políticas educacionales en gestión escolar implementadas en Chile, período 1990 - 2006 - Gustavo González García

orientada hacia la calidad. Tras esta valoración, las principales líneas de la política educacional fueron generar condiciones y dispositivos que permitieran instalar una lógica de mejoramiento continuo de la gestión, realizada en los establecimientos educacionales del país. Esto se dirigió a todos los actores del proceso educativo, organizándolos en torno a este fin y distinguiendo que hay roles diferenciados para cada uno de ellos: Ministerio, Sostenedores, equipos directivos, profesores, padres y apoderados.

Los diseños, esfuerzos y dispositivos más importantes de la política educacional en relación a la gestión escolar fueron:

- La creación en el año 2003 del Modelo de Calidad de la Gestión Escolar. Este recogió la experiencia acumulada en este ámbito a través de los Programas de Mejoramiento y de otras experiencias nacionales e internacionales relevantes, tales como la Guía de Autoevaluación de la Escuela y el instrumento ¿Qué tan bien está nuestro liceo? Orientaciones para la Autoevaluación. Para su creación se contó con el apoyo de Chile Calidad (Centro Nacional de la Productividad y Calidad CNPC), dependiente de CORFO, que otorga el Premio Nacional a la Calidad, en base al modelo de excelencia Malcolm Baldrige de gestión.

El Modelo identifica los procesos claves de la gestión de los establecimientos educacionales, los que se agrupan en cinco áreas generales (cuatro áreas de procesos: Liderazgo, Gestión Curricular, Convivencia y Recursos; y la de Resultados), subdivididas en I6 dimensiones y 57 elementos de gestión, permitiendo una evaluación específica de sus principales componentes.

- La implementación, a partir del año 2004, del Sistema de Aseguramiento de la Calidad de la Gestión Escolar(SACGE), éste se concibió como un conjunto coordinado de herramientas y recursos de apoyo al mejoramiento continuo de los procesos de gestión escolar, orientado a generar mejores condiciones institucionales para la obtención de resultados educativos, permitiendo su monitoreo y evaluación periódica (MINEDUC, 2005).

Los establecimientos educacionales que se incorporan al Sistema de Aseguramiento, desarrollaban un proceso interno de ejecución 
de cuatro fases: una Autoevaluación en base al Modelo de Calidad, una validación externa de la autoevaluación, el diseño de un Plan de Mejoramiento y una Cuenta Pública de los resultados de mejora.

- La elaboración de los Marcos de Actuación profesional para Directivos y docentes. El año 2003 se crea y difunde el Marco de la Buena Enseñanza y el año 2005 el Marco para la Buena Dirección. Estos instrumentos basados en el constructivismo pedagógico, la efectividad escolar, el liderazgo educativo, entre otros marcos conceptuales, entregan criterios de actuación profesional, que señalan la práctica de un buen profesor y de un buen director, para orientar, evaluar y mejorar la gestión de los profesionales de la educación.

Ambos marcos se acompañan de sistemas de evaluación del desempeño.

- El fortalecimiento del la Supervisión técnico-pedagógica, redefiniendo su rol y modernizando su gestión, con el objeto de que sea un real promotor y asistente de las lógicas de mejoramiento y de la gestión en los establecimientos. Al respecto, los supervisores y gestores de la supervisión comienzan entrenamientos en programas continuos de perfeccionamiento, sobre contenidos como el modelo de calidad y los marcos de actuación profesional. Además su gestión se enmarcó en el Ciclo de Asesoría, el cual consiste en el desarrollo de cuatro etapas sucesivas que los supervisores deben seguir en su relación de apoyo a los establecimientos (Diagnóstico y tres etapas relacionadas con un Plan anual de Asesoría: Planificación, Ejecución y Seguimiento y Evaluación). Este ciclo tiene como referencia el Modelo de Calidad. También se han creado dispositivos informáticos para modernizar la gestión de los supervisores, a través del desarrollo e implementación de un Sistema de Información y Monitoreo de la Supervisión (FOGES).

- La delegación del servicio de apoyo y asistencia técnica del Mineduc a Universidades o Centros Académicos y de Investigación, con el objetivo de provocar mejoramiento educativo en las escuelas que han sido favorecidas por las políticas educacionales desde I990, 
Desarrollo y análisis de las políticas educacionales en gestión escolar implementadas en Chile, período 1990 - 2006 - Gustavo González García

pero que son las más complejas y resistentes a obtener resultados y mejorar sus prácticas de gestión.

Esta política buscaba fortalecer la asistencia y supervisión del sistema educacional, entregando a los centros académicos la responsabilidad de asistencia técnica a los establecimientos educacionales prioritarios. El primero de estos proyectos fue la asesoría a 66 Escuelas Críticas de la Región Metropolitana, 2003-2005. Desde el año 2006 se desarrolló el proyecto de asistencia a escuelas y liceos prioritarios en varias regiones del país. Tras estos proyectos, la asistencia técnica en gestión escolar a los establecimientos fue parte esencial del diseño, por cuanto se entendía que lo central de estas asesorías era entregar capacidades institucionales a centros escolares que debían impulsar caminos de mejoramiento, cada vez más autónomos.

\section{c) El balance de las políticas educacionales en gestión escolar, período 2003-2006.}

Los cambios implementados en la política educacional desde el 2000, pero especialmente a partir de 2003, evidencian la centralidad de la gestión escolar como variable estratégica para elevar la calidad del sistema escolar chileno. La mayoría de estas políticas fueron evaluadas en estudios internos y externos del MINEDUC (Rodríguez y otros, 2005; Martinic y otros, 2006; González y otros, 2006; Raczynski y otros, 2006), sin embargo, falta cierta perspectiva de tiempo para evaluar impactos de largo plazo en la gestión de los centros escolares. A pesar de aquello, se pueden analizar las políticas en consideración de lo siguiente:

- La gestión escolar, de ser una política auxiliar a los Programas de Mejoramiento en los noventa, pasa a ser un pilar central de las políticas educacionales a partir del año 20003. Esto se basa en la concepción de que la calidad educativa se obtiene, tanto por una mayor responsabilización de los actores del sistema por sus actuaciones (gestión), como por el mejoramiento de las condiciones para asegurar la gestión de calidad.

- El Modelo de Calidad de la Gestión Escolar y los Marcos de 
Actuación Profesional, otorgaron una identificación de lo que es una buena escuela, una buena dirección y una buena enseñaza. Esto es fundamental para orientar procesos de mejoramiento, a partir del contraste de cada realidad escolar con el modelo.

En especial, el Modelo de Calidad y el SACGE respondieron al conocimiento acumulado de la investigación y experiencias nacionales e internacionales en calidad educativa, basados en movimientos y teorías como la Eficacia Escolar, Cambio Educativo, Liderazgo educacional, Mejora Escolar, Valor Agregado, entre los más significativos. Estos modelos y teorías tienen una ventaja con respecto a las ideas que fundamentaban la gestión escolar en la década anterior, debido a que teorizan desde lo educativo, a partir de las prácticas y experiencias escolares, constituyendo un saber teórico con estatus más original y contenido educacional. Esto favorece la mejor contextualización y difusión de estos modelos en las realidades escolares; para que los establecimientos se evalúen, se transformen y planifiquen su mejoramiento sobre la base de modelos basados en investigaciones y experiencias propiamente educacionales.

En el caso chileno, esto fue reforzado por una serie de estudios que desde fines de la década de los noventa identificaron y estudiaron experiencias nacionales de éxito educativo en contextos desfavorecidos, encontrándose centros escolares que presentan los mismos indicadores de eficacia conocidos a nivel internacional (Concha, 1996; Sancho, Arancibia y Smith, I997; Raczynski, Muñoz, Pérez y Bellei, 2004).

- Las políticas educacionales desde el 2000, no pierden de vista que la calidad educacional comienza con una buena gestión pedagógica en el aula, pero también depende de la buena gestión en todas las dimensiones de la organización escolar y se extiende más allá, en consideración de la buena gestión de los actores externos a la escuela: la gestión del apoyo y supervisión externa ministerial; el buen soporte de los sostenedores; la buena formación, capacitación y asistencia técnica que otorgan las universidades; el apoyo de los apoderados, etc. 
Desarrollo y análisis de las políticas educacionales en gestión escolar implementadas en Chile, período 1990 - 2006 - Gustavo González García

Estas Políticas educacionales en gestión escolar durante el período, se diseñaron para impactar a todo el sistema escolar público, aunque la preferencia estuvo en la implementación más decidida y focalizada en las escuelas más deficitarias.

Algunas dificultades o desafíos:

- Al igual que en la década de los noventa, la dificultad en la implementación de las políticas de gestión escolar a partir del 2000, está principalmente en lograr transformaciones educativas profundas, que impacten la cultura y desarrollo organizacional y no sólo conseguir cambios formales en los centros escolares.

En particular, el desafío estaba puesto en la capacidad de impactar y generar cambios de mejoramiento en establecimientos que manifestaban mayor resistencia a modificarse. Establecimientos que desde los noventa recibieron apoyo a través de los programas focalizados y, sin embargo, persisten durante el 2000, en sus malos resultados de aprendizaje y organizacionales (escuelas críticas y prioritarias). Estos centros mostraban realidades complejas, difíciles de cambiar por la sola disposición interna de estas organizaciones escolares, condición básica para que los modelos de calidad de la gestión tengan éxito, lo que implicaba como necesario una inteligente inducción externa del mejoramiento.

- Relacionado con lo anterior, un desafío teórico está en avanzar a la construcción y aplicación de modelos de asesoría educativa a centros escolares en contextos complejos. Provocar cambios educativos en establecimientos resistentes requiere de modelos apropiados a cada realidad escolar.

- Otro desafío de las políticas educacionales es la imprecisión de la gestión de calidad que se espera de los sostenedores educacionales. La investigación debe generar indicadores de calidad para este rol. Esto desde su tradicional función administrativa y financiera, pero también desde las funciones pedagógicas que algunos municipios están ejerciendo a través de la constitución de equipos técnicos municipales. 
- Otra dificultad de las políticas en gestión escolar, a partir del período, es la falta de conocimientos y modelos que permitan vincular de manera más consistente la gestión institucional con la gestión pedagógica. Es aceptado que el buen funcionamiento de una escuela influye en los buenos resultados que se logran en el aula, pero sobre esto hay mucho que profundizar desde la investigación, porque no es evidente la relación en algunos contextos escolares.

\section{Conclusiones}

La noción acerca de que un buen aprendizaje y una buena educación es el resultado no sólo de los procesos que se desenvuelven al interior del aula, en la relación de enseñanza-aprendizaje, entre profesor y alumnos; sino de manera importante, el desafío se coloca en juego a partir de procesos que están íntimamente ligados al primer ámbito, pero que ocurren al nivel inmediato y próximo de la organización escolar. Esta noción ha sido la motivación para que las políticas educacionales de los últimos veinte años en Chile, se ocuparan de crear condiciones que posibilitaran mejores formas de organizar y gestionar la escuela. Ya el proceso de descentralización educacional, llevado a cabo durante los años ochenta, descansaba en esta noción; con la pretensión de que una administración más al nivel local de los asuntos escolares, lograría mayor eficacia educativa.

Este artículo, describió y analizó el desarrollo general de estas políticas educacionales en gestión escolar, durante el período I9902006. Para su construcción se consideraron artículos y libros de los propios diseñadores de las políticas. También se analizaron documentos, orientaciones e instrumentos oficiales, elaborados para la implementación de la política. Desde una perspectiva crítica, se revisaron las investigaciones e informes de evaluación, sobre la aplicación de estas políticas, que realizaron instituciones académicas independientes.

A manera de conclusión, el proceso que más interesa destacar aquí, es que en el diseño de las políticas educacionales, la gestión escolar 
Desarrollo y análisis de las políticas educacionales en gestión escolar implementadas en Chile, período 1990 - 2006 - Gustavo González García

transitó desde una valoración auxiliar en los noventa, hacia una posición estratégica y central, a partir del 2000.

En los noventa, se concebía una buena gestión escolar como aquel soporte institucional adecuado a las transformaciones de las prácticas curriculares y pedagógicas. Existiendo otras prioridades de las políticas educacionales, como la Reforma curricular, el desarrollo profesional docente, la mejora en las condiciones de funcionamiento del sistema, etc. la direccionalidad de la política iba desde lo macro del sistema escolar a lo micro (aula), sin ocuparse significativamente de la gestión del nivel medio del sistema; como la gestión en la escuela, la administración local, la gestión supervisora o la gestión de asesoría entre el mundo académico y los establecimientos educacionales.

Comenzando el nuevo siglo, el paso a la centralidad de la gestión escolar comienza con la contemporaneidad de distintos procesos. Por una parte, desde la autoridad educacional se valora crecientemente la dependencia entre la eficacia de las políticas de mejoramiento pedagógico, con las condiciones de recepción en los establecimientos educacionales. Desde la evaluación de las políticas y programas de mejoramiento educativo, se concluía de forma similar -evaluaciones nacionales e internacionales (OECD)-, que siendo bien orientado el diseño de la política educativa, ésta no transformaba natural y rápidamente la realidad escolar como se deseaba, quedando en evidencia que estas transformaciones dependían decisivamente, entre otros factores, de la calidad de implementación de la política y de las condiciones de recepción y gestión de los actores de la escuela. Esto llevó a relevar estratégicamente la gestión de los actores intermedios y finales del sistema; algunos relegados a un rol menos determinante durante los noventa; supervisores, sostenedores, directivos, organismos académicos y de asesoría. Otros, a quienes se les redefinía su rol y gestión a partir de elementos no sólo didácticos y curriculares: los docentes.

Paralelamente, desde los conocimientos teóricos y de su impacto en las políticas, se transitó desde concepciones que apropiaban los modelos organizacionales y el management moderno, hacia concepciones de 
la institución escolar como organización singular y compleja. En este proceso, fueron determinantes los estudios de escuelas eficaces; los movimientos de mejora y cambio educativo; la conceptualización de la calidad educacional y los modelos de evaluación educativa que de aquí surgieron; la identificación de estándares e indicadores de calidad para actores y organizaciones escolares; y los sistemas de acreditación o certificación de calidad escolar, aseguramiento y evaluaciones de desempeño.

Todos estos elementos conceptuales fundamentan teóricamente las políticas de gestión escolar del año 2000 en adelante.

Desde el punto de vista del impacto de las políticas en gestión escolar, durante los noventa el carácter auxiliar que tuvo la gestión escolar no permitió provocar cambios significativos y a nivel del sistema, en este ámbito. Sin embargo, en ningún caso se puede hablar de una década perdida, debido a que existieron logros significativos y una continuidad institucional, en donde lo realizado antes, sirvió de base para el desarrollo posterior.

Desde el año 2003 la gestión escolar pasa a ser un elemento central de la Reforma educacional, representando una variable ampliamente valorada y estratégica para alcanzar mejores procesos y resultados escolares.

El gran desafío de interés público que tienen las políticas de gestión escolar, a partir del 2003, es lograr la transformación y mejoramiento de las prácticas escolares en los establecimientos con mayores dificultades y bajos rendimientos, los cuales no han mejorado al nivel de los esfuerzos y recursos puestos en ellos desde 1990. Las políticas de gestión escolar están especialmente dirigidas, en conjunto con otras reformas del sistema escolar, a colaborar en este proceso de equidad, elevando los resultados escolares de todo el sistema, a partir de la nivelación de la calidad bacia arriba (centros escolares con bajos rendimientos). Sin embargo, también están orientadas a impactar indirectamente en todos los establecimientos educacionales, enfocando el progreso educativo a partir de lo que se concibe como 
Desarrollo y análisis de las políticas educacionales en gestión escolar implementadas en Chile, período 1990 - 2006 - Gustavo González García

una buena gestión de los actores escolares y a través de la creación de contextos organizacionales e ínter-organizacionales dirigidos al mejoramiento.

Con todo, uno de los principales riesgos del papel estratégico que toman las políticas de gestión escolar a partir de este siglo, es su sobrevaloración como variable determinante en la elevación de los resultados escolares del sistema. El mejoramiento de la calidad del sistema educacional pasa por muchos factores conocidos y discutidos: entre otros: mayor financiamiento; problemas estructurales de administración educativa comunal y lógicas de mercado; calidad en la formación inicial de docentes; pertinencia y relevancia del curriculum (OCDE, op. Cit: Consejo Asesor Presidencial para la Calidad de la Educación, 2006). Por lo que, cualesquiera sean los resultados en calidad que obtenga el sistema escolar a partir del año 2006, positivos o negativos: desde el punto de vista de la lógica pública, las políticas educacionales con mayor visibilidad estratégica, serán las que se sometan a examen, por sobre otras que se implementen -o que no se bayan implementado-. Esto puede llevar a una evaluación -y a sus consecuencias-más política que técnica, sobre algunas o todas las políticas en gestión escolar, implementadas desde el año 2000 en adelante.

\section{Bibliografía}

I. Alvariño, C.; Arzola, S.; Brunner, J.J.; Recart, M.; Vizcarra, R. (2000). "Gestión Escolar: un estado del arte de literatura". Revista Paideia 29 (pp.: I5 - 43). Universidad de Concepción, Chile. Disponible en: http://www. educacion.es/cide/espanol/investigacion/rieme/documentos/files/ alvarino/alvarino.pdf.

2. Asesorías para el Desarrollo (2000). Evaluación del Programa de Mejoramiento de la Calidad de las Escuelas Básicas de sectores pobres P-900, Informe Final, Santiago de Chile.

3. Astudillo, E.; Astudillo, O.; Cuadra, G.; Guerrero, G.; Torres, M. y Vaccaro, L. (I996). Manual para Equipos de Gestión Escolar, Ministerio de Educación, Santiago de Chile.

4. Concha, Carlos (1996). Escuelas efectivas en Chile: estudio de 32 escuelas exitosas en logros académicos y de alta vulnerabilidad, Tesis Magíster Educacional, Pontificia Universidad Católica de Chile, Santiago. 
5. Consejo Asesor Presidencial para la Calidad de la Educación (2006). Informe Final del Consejo Asesor Presidencial para la Calidad de la Educación, Santiago de Chile.

6. Filp, J. C.; Cardemil, S.; Donoso, J.; Torres, L.; Diéguez, y Schiefelbein (198I/I982). "La Escuela ¿Cómplice del fracaso Escolar?”, en: Revista de Tecnología educativa. Santiago, OEA, № 4, Vol. 7 (pp.: 340 - 358).

7. Filp, J. et al. (I99I). Evaluación del Programa de las 900 Escuelas. Resumen Ejecutivo. CIDE, Santiago de Chile.

8. Gairín, Joaquín (1996). La organización escolar: contexto y texto de actuación, Ed. La Muralla, España.

9. García Huidobro, Juan Eduardo; Sotomayor, Carmen (2003). "La Centralidad de la Escuela en la Política Educativa”, en Políticas Educacionales en el Cambio de Siglo, Cristián Cox (editor), Editorial Universitaria, Santiago de Chile.

I0. González, G. et. al. (2006), Servicios para la Difusión de las Acciones y Resultados del Sistema de Aseguramiento de la Calidad de la Gestión Escolar a Autoridades Municipales de Educación, Transversal, Santiago de Chile.

II. Himmel, E.; Maltes; Majluf. et al. (1984). Análisis de la influencia de factores alterables del proceso educativo sobre la efectividad escolar. Pontificia Universidad Católica de Chile, Santiago.

I2. Larraín, Trinidad (2002). "La Propuesta de Gestión Educativa”, en El Programa de las 900 escuelas: trayectoria de una década. Ministerio de Educación Chile, Santiago de Chile.

13. Larraín, Trinidad (2002b.) Hacia una Gestión Autónoma y centrada en lo Educativo. Propuesta del Programa de las 900 Escuelas, Ministerio de Educación, Santiago de Chile.

I4. Lavín, S. y Del Solar, S. (I997). El Proyecto Educativo Institucional como berramienta de construcción de identidad, MINEDUC, Santiago de Chile

I5. Martinic, S.; Cádiz, J.; Madrid, A.; Ruz, M.A.; Fernández, M. (2005.) Evaluación del Programa de las 900 Escuelas. Trienio 2001 2003. Informe Final. Pontificia Universidad Católica de Chile, Facultad de Educación, Santiago de Chile.

I6. Martinic, S.; Volante, P.; Huepe, D. y González, G. (2006). Sistematización, Comparación y Análisis de los Registros ingresados por los Supervisores al Sistema de Información y Monitoreo de la Supervisión (FOGES), Pontificia Universidad Católica de Chile, Santiago.

17. Ministerio de Educación (2000). Guía de Autoevaluación de la Escuela, Ministerio de Educación, Santiago de Chile. 
Desarrollo y análisis de las políticas educacionales en gestión escolar implementadas en Chile, período 1990 - 2006 - Gustavo González García

18. Ministerio de Educación (2005), Calidad en todas las Escuelas y Liceos. Sistema de Aseguramiento de la Calidad de la Gestión Escolar. MINEDUC, Serie Bicentenario, Santiago de Chile.

19. OCDE (2004). Revisión de Políticas Nacionales de Educación: Chile, Centro para la cooperación con los países no miembros de la OCDE, Santiago, Chile.

20. Raczynski, D. et al. (2002). Análisis de la Oferta de Programas e Iniciativas del Sector Público y Privado desde los Establecimientos Educacionales, Asesorías para el Desarrollo, Santiago de Chile.

2I. Raczynski, Muñoz, Pérez y Bellei (2004). ¿Quién dijo que no se puede? Escuelas Efectivas en Sectores de Pobreza. UNICEF, Santiago de Chile.

22. Raczynski, D. et al. (2006). Sistematización y evaluación del Plan de asistencia técnica a escuelas críticas de la Región Metropolitana, Asesorías para el Desarrollo, Santiago de Chile.

23. Rodríguez, B. et al. (2005). Sistematización Planes de Mejoramiento Sistema de Aseguramiento de la Calidad de la Gestión Escolar 2004. Informe Final, Universidad Austral, Valdivia.

24. Sancho, A.; Arancibia, V.y Smith, P. (1997). Experiencias Educacionales Exitosas. Instituto Libertad y Desarrollo, Santiago de Chile. 